\title{
A Method Based on Prior Information of Feature Points Registration for SAR Images
}

\author{
Dekun $\mathrm{Li}^{1, \mathrm{a}}$, Pingyan Shi ${ }^{1, \mathrm{~b}}$, Xiaomin $\operatorname{Tan}^{1, \mathrm{a}}$ \\ ${ }^{1}$ Xi'an Institute of Space Radio Technology, Xian 710100, China;

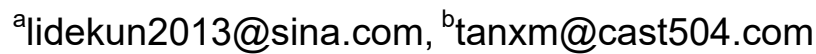

Keywords: SAR images, Sequence registration, Prior information, SIFT algorithm.

\begin{abstract}
The concept of moving target detection is monitoring and detecting the moving target in the target area, achieving the goal of monitoring the target area all-day and all-weather. During the process of monitoring, the multi-frame SAR images which get from the same scenario need to be registered. The Range Doppler equations are used to obtain the location model of SAR images in this paper, combing the SIFT algorithm with the prior information. Firstly, the SIFT is used to extract the feature points. Secondly, the prior information is used to achieve the regional division of the feature points. Finally, the nearest neighbor matching rule is used to match the targets in the candidate target region. The prior information of SAR images is fully used in this method. In this way, the matching search range is reduced and the efficiency of matching algorithm is improved. Real data simulations prove that this algorithm is feasible.
\end{abstract}

\section{Introduction}

SAR imaging technique is used for imaging stationary targets, and GMTI technique is used for detecting and tracking moving targets. With these two technologies, SAR-GMTI system cannot only image for regional targets, but also detect moving target on the ground and form the target track. The realization of SAR-GMIT requires Sequence registration of multiple frames of SAR images in the same scene [1]. Currently, SAR image registration [2] is divided into two methods: gray information-based and features-based. Registration based on features extracts the image feature information to describe the characteristics. Then finds match feature using matching criteria and search methods to complete image registration [3].

Lowe proposed SIFT feature point matching algorithm [4, 5] in 1999;Wang J, Pi Y, Cao Z proposed a registration using extraction area features [6] in 2008; Jean M.M proposed ASIFT algorithm [7] in 2009. Mean time, Suri combined the SIFT algorithm with mutual information [8, 9]; Shanhu Wang and Hongjian You proposed a algorithm that match images roughly to eliminate the effects of image rotation and scale, then realize precise registrationusing Harris SAR image feature extraction algorithm [10] in 2012; Baoshang Zhang, Tian Zheng and Weidong Yan proposed a SAR image registration method based on the divided regions [11]; Guiqin Xia combined the corner match with prior information [12] in 2014. Registration method based on the point feature is simple and convenient. However, speckle noise in SAR image can lead to a lot of feature points extracted and it will increase the amount of arithmetic operations. How to reduce the computation and improve the robustness of the algorithm is a key research direction of registration algorithm based on the feature point. This article combines SIFT algorithm with the prior information. It divides the feature points into different region using the prior information to avoid the global feature matching. As a result, the SAR image registration algorithm efficiency is improved.

\section{SIFT algorithm}

Through a Gaussian differential function, the SIFT algorithm generates a scale space, and selects local extreme point as the candidate feature point, then removes the unstable low contrast and edge response points, and precisely positions feature points [13] [14]. The flowchart of SIFT algorithm for image registrations shown in Fig 1. 


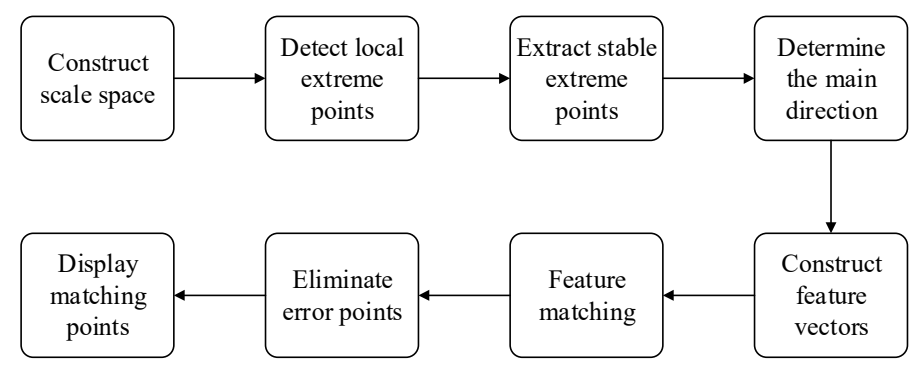

Fig. 1 1SIFT algorithm flowchart

Image data is sampled down, and the image pyramid is constructed. Gaussian convolution kernel can achieve the scale transformation [15]. Two-dimensional image can be formed as image scale space with the convolution to different kernel Gaussian filters. As follows:

$$
L(x, y, \sigma)=G(x, y, \sigma) \otimes I(x, y)
$$

Wherein, $I(x, y)$ is an input image, $G(x, y, \sigma)=\frac{1}{2 \pi \sigma^{2}} e^{-\left(x^{2}+y^{2}\right) / 2 \sigma^{2}}$ is the Gaussian kernel function, $\sigma$ is a variable kernel.

Extreme point of Gaussian Laplace can provide stable characteristic for the image [5].

$$
\frac{\partial G}{\partial \sigma}=\sigma \nabla^{2} G \approx \frac{G(x, y, k \sigma)-G(x, y, \sigma)}{(k-1) \sigma}
$$

Gaussian Laplace function can be approximated by Gaussian differential function. And the extreme points can be obtained by Gaussian differential function.

$$
\begin{aligned}
D(x, y) & =[G(x, y, k \sigma)-G(x, y, \sigma)] \otimes I(x, y) \\
& =L(x, y, k \sigma)-L(x, y, \sigma)
\end{aligned}
$$

Gaussian function fits to the three-dimensional quadratic function to achieve the positioning accuracy of the feature points. Gaussian differential function can be expanded by Taylor formula on candidate extreme points, as follows:

$$
D(X)=D\left(X_{0}\right)+\frac{\partial D^{T}}{\partial X}\left(X-X_{0}\right)+\frac{1}{2}\left(X-X_{0}\right)^{T} \frac{\partial^{2} D}{\partial X^{2}}\left(X-X_{0}\right)
$$

Wherein, $X_{0}=\left(x_{0}, y_{0}, \sigma_{0}\right)^{T}$ and $X=(x, y, \sigma)^{T}$ are the vectors of the space position and the scale respectively of the extreme points and the candidate points. $D\left(X_{0}\right)$ is the Gauss differential value of the candidate feature points. Take the derivative of the equation with respect to $\left(X-X_{0}\right)$, and set its first-order partial derivative equal to zero, then adjust the position of the extreme points. As follows:

$$
D(X)=D\left(X_{0}\right)+\frac{1}{2} \frac{\partial D^{T}}{\partial X} \Delta X
$$

Detecting extreme point of differenced Gaussian has a strong edge response. There is a proportional relationship between the principal curvatures of the edge points and its eigenvalue of the Hessian matrix. Calculate the eigenvalue of the Hessian matrix to determine whether it is the stable point.

Lowe proposed the method to construct feature points' descriptors using the gradient distribution of the feature points' neighboring pixels. Gradient magnitude and direction of pixel $(\mathrm{x}, \mathrm{y})$ are expressed as follows:

$$
\begin{aligned}
& M(x, y)=\sqrt{(L(x+1, y)-L(x-1, y))^{2}+(L(x, y+1)-L(x, y-1))^{2}} \\
& \theta(x, y)=\tan ^{-1}((L(x, y+1)-L(x, y-1)) /(L(x+1, y)-L(x-1, y)))
\end{aligned}
$$

The gradient distribution is counted by the center pixel of the feature points. Histogram about the gradient direction is divided into 36 columns, and the peak value indicates as the main direction of the feature points. Rotate the axis of the image to the main direction of the feature points. Use Gaussian weighted principal to calculate the accumulated value of the pixel in the window in each direction of the gradient. Neighborhood window is divided into 16 sub-blocks of $4 \times 4$, and statistic the accumulated value of gradient histogram of 8 directions in each sub-block, then generate a seed 
point. Statistical formula is as follows:

$$
h_{r(l, m)}(k)=\sum_{x, y \in r(l, m)} M(x, y)\left(1-\left|\theta(x, y)-c_{k}\right| / \Delta_{k}\right), \quad \theta(x, y) \in \operatorname{bin}(k)
$$

Wherein, $c_{k}$ is the center of direction column, $\Delta_{k}$ is the width of the column direction and (x,y) is the coordinate of the pixel of sub-block $r(l, m)$. The feature vector of the feature points is constructed by $4 * 4$ sub-block histogram, forming a 128-dimensional vector:

$$
u=\left(h_{r(1,1)}, \mathrm{L} h_{r(1, m)}, \mathrm{L}, h_{r(4,4)}\right)
$$

The minimum Euclidean distance can be used as the similarity metric criterion in the 128-dimensional feature vectors extracted from the SIFT algorithm. Euclidean distance between two feature points can be expressed as for ( $\mathrm{N}$ is the dimension of feature vectors):

$$
\operatorname{dis}(i, j)=\sqrt{\sum_{k=1}^{N}\left(u_{i}(k)-u_{j}(k)\right)^{2}}
$$

Similar structures existing in the image will result in error matching points which scattered in the similar structures. The false match can be eliminated by the ransac algorithm, achieving the exact match.

\section{SAR image registration combined with prior information}

Range-Doppler model is based on the squint SAR imaging and the characteristics of Doppler frequency shifting [12] [16]. Geometry model of SAR imaging is shown in Fig 2. V represents carrier aircraft velocity, $\mathrm{H}$ represents the height, $\mathrm{O}^{\prime}$ is the ground projection of the starting position, $\mathrm{Y}$-axis is the direction of carrier aircraft flight and $\mathrm{Z}$-axis is perpendicular to the ground. So the scene coordinate system is $\mathrm{O}^{\prime}-\mathrm{XYZ}$.

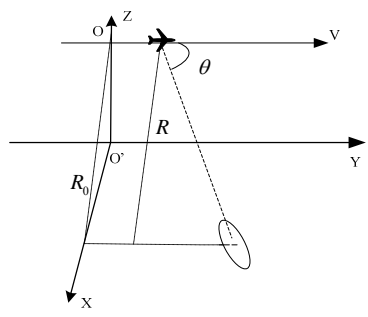

Fig. 2 geometry model of SAR imaging

Target distance is determined by the position of the received echo delay. The slant distance [17] to the j-th pixel of range direction in SAR image is as follows:

$$
R=R_{\min }+\frac{c}{2 f_{s}} \cdot j
$$

The azimuth location of target is determined by the Doppler characteristics and $f_{d}$ represents Doppler frequency of the target. As follows:

$$
f_{d}=2 \frac{v}{\lambda} \cos \theta
$$

$R_{\min }$ is the shortest distance from the center of the antenna to the swath. $f_{s}$ is the A / D sampling frequency, and $\theta$ is the angle between the antenna center radial and aircraft flight direction. We can get the coordinates of the scattering ground point corresponding to pixel $(i, j)$ under scene coordinate system:

Carrier aircraft flight path is not necessarily true north direction, so we need to change the scene coordinates to carrier aircraft geographic coordinates. In order to achieve the goals' absolute positioning, the aircraft geographic coordinates should be converted tothe geodetic coordinates. The target positioning process of the SAR image can be shown in Fig 3. 


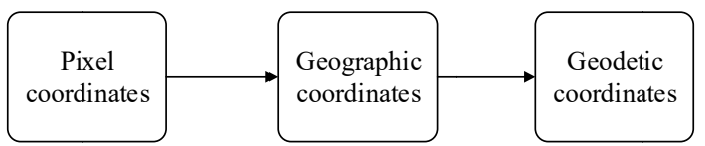

Fig. 3 SAR target location flowchart

In usual registration method, the shortest Euclidean distance criterion is used to search for all the extracted feature points globally. In this paper, the imaging area geographic location is determined by the INS data and imaging parameters. We can make use of the SAR image localization model to find geodetic coordinates corresponding to the pixel coordinates. Then the corresponding coordinates of match point pixel in the registered SAR image can be retrieved. The candidate matching area is determined by the estimated matching point's position as the center to reduce the scope of the search feature points and find a match point finally.

The process of SAR image registration algorithm based on prior information of feature points is divided into four steps. Firstly, get the location of carrier aircraft and calculate the Geodetic coordinates of $\mathrm{O} 1$ and $\mathrm{O} 2$ according to the INS data and imaging parameters. Secondly, use the Range-Doppler model and prior information to determine the position of the reference feature points under the geographic coordinate system. Thirdly, convert the above geographic coordinate to another geographic coordinate. Then the geographic coordinate uses Range-Doppler model to convert it to pixels point coordinates in the registration image. Finally, select the matching point by the nearest neighbor matching rule from the corresponding pixel coordinates in the registration image. The flow chart of SAR image registration algorithm based on prior information is shown in Fig 4.

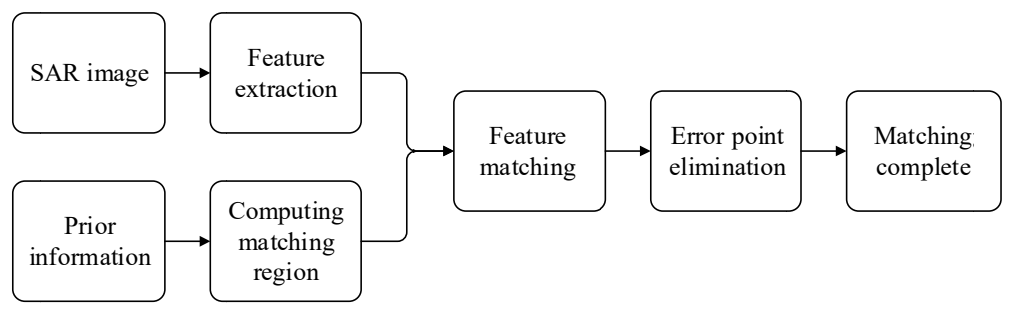

Fig. 4 Flowchart of image registration methodcombined with SAR image prior information

\section{Simulation analysis}

The analysis for the SIFT algorithm which is combined with prior information through measured data is as follows:

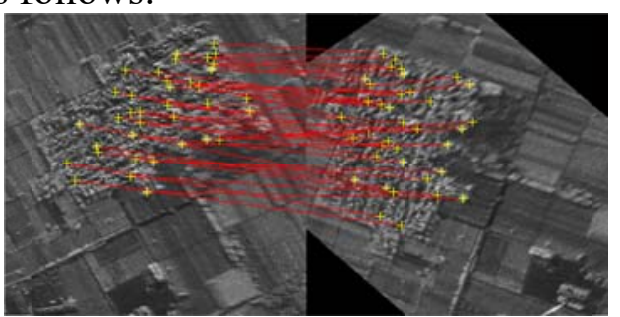

Fig 5 Feature point matching results

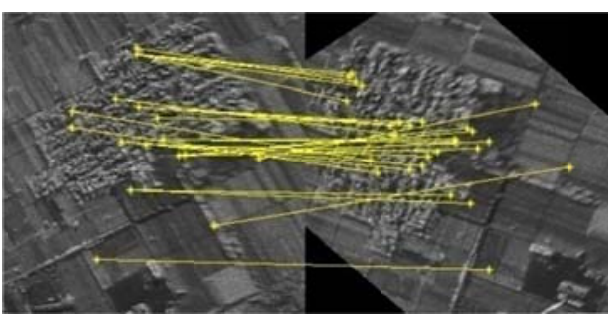

Fig 6 Feature point matching results

Experiment 1, Air borne low-resolution image is registered through the above algorithm. The left is reference image and the right is the generated image with different squint. The results are as follows: Fig 5 (global search), Fig 6 (local search with prior information).

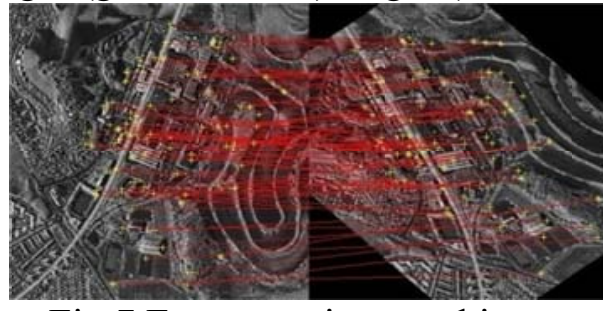

Fig 7 Feature point matching results

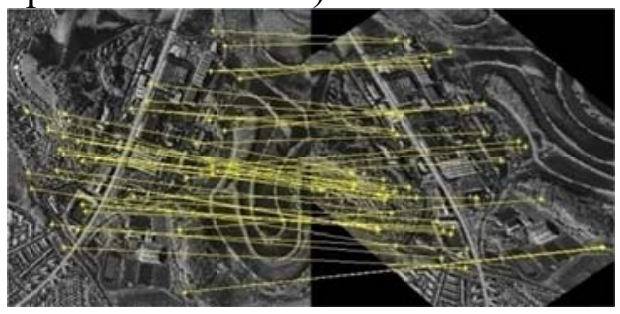

Fig 8 Feature point matching results

Experiment 2, Air borne high-resolution image is registered through the above algorithm. The 
left is reference image and the right is the generated image with different squint. The results are as follows: Fig 7 (global search), Fig 8 (local search with prior information) .

Table 1 Experimental comparison between global search and local search

\begin{tabular}{ccccccc}
\hline $\begin{array}{c}\text { registration } \\
\text { algorithm }\end{array}$ & $\begin{array}{c}\text { Feature point } \\
\text { numbers (Fig } \\
\text { 5/Fig 6) }\end{array}$ & $\begin{array}{c}\text { Match } \\
\text { time /s }\end{array}$ & $\begin{array}{c}\text { Error match } \\
\text { point/ } \\
\text { Matchpoint }\end{array}$ & $\begin{array}{c}\text { Feature point } \\
\text { numbers (Fig } \\
7 / \text { Fig 8) }\end{array}$ & $\begin{array}{c}\text { Match } \\
\text { time /s }\end{array}$ & $\begin{array}{c}\text { Error } \\
\text { matchpoint/ } \\
\text { Match point }\end{array}$ \\
\hline global search & $109 / 118$ & 0.11 & $0 / 37$ & $332 / 334$ & 0.28 & $5 / 80$ \\
\hline local search & $109 / 118$ & 0.03 & $0 / 27$ & $332 / 334$ & 0.04 & $0 / 41$
\end{tabular}

The conclusion can be obtained through the experiments. The matching search range of the feature points is greatly reduced with the method which is combined with SAR imaging prior information to determine the candidate match area. Meantime, the matching speed is improved and the matching rate has a more obvious advantage on the rapidly increasing of the feature points.

\section{Summary}

SAR image registration is critical to applications of SAR data. It has deep influence on SAR image correction, target detection, image change detection, DEM extraction and so on. Therefore, the high efficiency and precision of the SAR image registration have been put forward. In this paper, the Range-Doppler Equation has been used to derive SAR image localization model and the theoretical basis of image registration has been introduced. Then, combined with SAR prior information, the extracted feature points are located by using parameters of SAR image and platform navigation system, and the registration image candidate matching point regions are determined. Finally, matching points are searched in the candidate area. This method takes full advantage of prior information of SAR imaging to avoid global search. Proved by real data, the algorithm's precision and efficiency are improved.

\section{References}

[1]. Shichao Zheng, Yabo Liu, Hongjun Song, et al. A Research on Moving Target Trajectory Simulation and Tracking in Wide Area Surveillance Ground Moving Target Indication Mode [J]. Journal of Electronics \& Information Technology, 2013, 35(9): p. 2108-2113.

[2]. Qiaoyan Li. Research of scene matching algorithm based on SAR image [D]. Xi'an Electronic and Science University. 2011.

[3]. Ying $\mathrm{Xu}$, Yan Zhou. Review of SAR image registration method [J]. Geospatial Information. 2013, 11(3): p. 63-66.

[4]. Lowe D.G. Obeject recognition from local scale-invariant features [C]. Proceedings of International Conference on Computer Vision. Kerkyra, 1999, p. 1150-1157.

[5]. Lowe D.G. Distinctive image features from scale-invariant features [J]. International Journal of Computer Vision. 2004, 60(2), p. 91-110.

[6]. Wang J, Pi Y, Cao Z. Level set method for SAR image coregistration [J]. IEEE Geoscience and Remote Sensing Letters. 2008, 5(4): p. 615-619.

[7]. Morel J M, Yu G S. ASIFT: A New Framework for Fully Affine Invariant Image Comparison [J]. IEEE Trans Pattern Analysis and Machine Intelligence. 2009. 23(2): p. 1289-1293.

[8]. SahiSuri, Peter Schwind. Combining mutual information and scale invariant feature transform for fast and robust multisensor SAR image registrantion [C]. Proceedings of 75th ASPRS Annual Conference. Baltimore, USA, 2009. p. 145-150. 
[9]. SahiSuri, Peter Schwind. Modifications in the SIFT operator for effective SAR image matching [J]. International Journal of Image and Data Fusion, 2010 1(3):243-256.

[10]. Wang Shanhu, You Hongjian. BFSIFT: A novel method to find feature matches for SAR image registration [J]. IEEE Geoscience and Remote Sensing Letters. 2012, 9(4), p. 649-653.

[11]. Baoshang ZHANG, Zheng TIAN, Wei-dong YAN. An SAR Image Registration Algorithm Based on Segmentation-derived Regions [J]. Chinses journal of engineering mathematics. 2011, 28(1), p. 7-14.

[12]. Guiqin Xia. Research on image sequence alignment method for radar of WAS SAR-GMTI large angle of view [D]. Xi'an Electronic and Science University. 2014.

[13]. Daoyin Wang. Research on image registration algorithm based on SIFT [D]. University of Science \& Technology China. 2011.

[14]. Jinjian $\mathrm{Lu}$. Research on multi source remote sensing image registration based on feature [D]. National university of defense technology. 2008.

[15]. Lindeberg, Tony. Scale-space theory: A basic tool for anslysingstructurea at different scales [J]. Journal of Applied Statistics. 1994, 219(2),p. 224-270.

[16]. John C. Curlander. Location of space-borne SAR imagery [J]. IEEE Transaction on Geoscience and Remote Sensing. 1982, 20(3), p.359-364.

[17]. Zhangquan ZHANG, Wenfeng SUN. Analysis of Air-borne SAR Image Location Precision [J]. Journal of air force radar academy. 2007, 21(4), p. 263-265. 